Check for updates

Cite this: Phys. Chem. Chem. Phys., 2018, 20, 17380

Received 2nd May 2018 Accepted 7th June 2018

DOI: $10.1039 / \mathrm{c} 8 \mathrm{cp} 02802 \mathrm{~h}$

rsc.li/pccp

\section{Bilayers of Janus WSSe: monitoring the stacking type via the vibrational spectrum}

\author{
A. Kandemir (iD a and H. Sahin (D) bc
}

\begin{abstract}
Motivated by the recent successful synthesis of Janus type single layers of transition metal dichalcogenides, we investigate the stability, vibrational and electronic properties of the Janus single layer structure of WSSe and its bilayers by means of density functional theory. The structural and vibrational analysis show that the Janus single layer of WSSe forms a dynamically stable structure in the $2 \mathrm{H}$ phase. Owing to its noncentrosymmetric structure, the Janus WSSe single layer has two in-plane (E) and two out-of-plane (A) Raman active phonon modes. The eigen-frequencies of the prominent Raman active modes are calculated to be 277 (A) and 322 (E) $\mathrm{cm}^{-1}$. Similar to single layer $\mathrm{WS}_{2}$ and $\mathrm{WSe}_{2}$, Janus WSSe is a direct band gap semiconductor that has two electronically different faces. In addition, the possible bilayer stacking orders of the Janus WSSe single layers are investigated. It is found that there are 3 stacking types of bilayer Janus WSSe and each stacking type has distinctive Raman characteristics in its vibrational spectrum. Our results show that thanks to the vibrational characteristics, which stem from the distinctive interlayer interactions at different sides, the stability and stacking types of the bilayer of WSSe Janus structure can be monitored.
\end{abstract}

\section{Introduction}

Recent advances in the experimental synthesis of two dimensional (2D) materials have led to the existence of a variety of stable ultrathin crystals. ${ }^{1-5}$ In terms of technological applications, characterization of these ultra-thin materials at the nanoscale is essential. For the determination of the ground state phase, atomic composition, thickness and stacking type of ultra-thin crystals, vibrational characteristics obtained from Raman spectroscopy and theoretical tools are of importance. ${ }^{6}$

Ultra-thin transition metal dichalcogenides (TMDs), a family of $2 \mathrm{D}$ materials, have received extraordinary attention owing to their remarkable electronic properties in nano-devices. ${ }^{7-15}$ The members of the TMDs, tungsten dichalcogenides, $\mathrm{WS}_{2}$ and $\mathrm{WSe}_{2}$ are the pillars of low dimensional materials for photonic applications. $^{7,16}$ In the last decade, atomically thin tungsten dichalcogenides have been produced uniformly, in a highly scalable manner, with a size of large-area, and with high-quality on different substrates and they have been proposed as flexible low-power optoelectronic devices. ${ }^{17-21}$ The stable atomic structure, and easily tunable electronic and optical properties of tungsten

\footnotetext{
${ }^{a}$ Department of Materials Science and Engineering, Izmir Institute of Technology, 35430, Izmir, Turkey.E-mail: alikandemir@iyte.edu.tr

${ }^{b}$ Department of Photonics, Izmir Institute of Technology, 35430, Izmir, Turkey. E-mail: hasansahin@iyte.edu.tr

${ }^{c}$ ICTP-ECAR Eurasian Center for Advanced Research, Izmir Institute of Technology, 35430, Izmir, Turkey
}

chalcogenides promise enormous opportunities for their further applications in future device technologies.

In addition to the superior features of TMD single layers, bilayers of TMDs exhibit significant properties for devices in different areas with an effortless scalable fabrication process. ${ }^{22-24}$ By the van der Waals assembly of TMDs, Lee et al. reported the characterization of the electronic properties of an atomically thin $\mathrm{p}-\mathrm{n}$ junction device. ${ }^{22}$ Hong et al. observed the ultrafast charge transfer in a $\mathrm{MoS}_{2} / \mathrm{WS}_{2}$ heterostructure. ${ }^{23}$ Tunable interlayer coupling in a $\mathrm{MoS}_{2} / \mathrm{WS}_{2}$ bilayer with vacuum thermal annealing was shown by Tongay et al. ${ }^{25}$ van der Waals heterostructures of molybdenum and tungsten chalcogenides with different configurations have been produced as photo-voltaic devices that possess functionalities, and spectacular electronic and optoelectronic properties. ${ }^{26-28}$ In addition to the electronic and optical properties, the thickness-dependent vibrational properties of TMDs have been investigated from the monolayer to bulk for providing a unique fingerprint and rapid identification. ${ }^{12,29-31}$ External effects such as strain and temperature on the vibration properties of TMDs have been studied widely. ${ }^{6,32-35}$ Strain effects on the electronic and vibrational properties of $\mathrm{WS}_{2}$ monolayers were analyzed by Wang et al. and Raman spectroscopy results revealed noticeable Raman-shifts and splitting in the in-plane optical mode under tensile strain. ${ }^{35}$

Very recently, a different method was used for tailoring the design of 2D materials at the atomic level; the formation of Janus crystals. Besides computational studies, ${ }^{36,37}$ the experimental studies of Lu et al. ${ }^{38}$ and Zhang et al. ${ }^{39}$ have shown the modification of the $\mathrm{MoS}_{2}$ structure as S-Mo-Se sandwiched 
layers, and hence, synthesized a Janus MoSSe single layer. Additionally, it was shown that the dynamical and electronic properties differ in the Janus structure compared to its analogs. In addition to the experimental realization of the Janus structure, the larger lattice constant and relatively weak bonds of Se atoms with transition metal atoms in a $\mathrm{WSe}_{2}$ single layer enable the easy synthesis of Janus WSSe single layers via the well-known sulfurization process. Also, very recent work of Er et al. ${ }^{40}$ has shown the hydrogen evolution reaction catalytic activity of Janus 2D TMDs and they presented the Janus WSSe single layer as a promising candidate.

In this paper, we study the structural, vibrational and electronic properties of a $2 \mathrm{H}$-stable Janus single layer structure, WSSe. Furthermore, the stacking orders of the bilayer of Janus WSSe structure are studied. Possible stacking types of the bilayer are determined due to the ground state energies. The distinct vibrational properties between stacking orders of the bilayers are revealed. Herein, we show the possibility to monitor the stacking orders of the bilayers of Janus WSSe structures via vibrational properties; thus, Raman spectroscopy can be utilized to identify the stacking type of the bilayers of Janus structures. This paper is organized as follows: the computational methodology is given in Section II. The structural, electronic and vibrational properties of single layer WSSe are presented in Section III. The structural and vibrational characteristics of the bilayers of Janus WSSe stacking types are presented in Section IV. Section V is devoted to the conclusion.

\section{Computational methodology}

For the determination of the ground state atomic structure, the electronic charge density and the forces acting on each atom of the Janus WSSe single layer, the Vienna $a b$ initio Simulation Package (VASP) ${ }^{41,42}$ implemented with the-plane-wave basis projector-augmented wave (PAW) method was used. For the exchange-correlation part of the calculations, the generalized gradient approximation (GGA) in the Perdew-Burke-Ernzerhof (PBE) form was applied. ${ }^{43}$ The van der Waals (vdW) correction to the GGA functional was inserted by using the DFT-D2 method of Grimme. ${ }^{44}$ To cope with the underestimation of the band gap given by pure GGA within the inclusion of spin-orbit coupling (SOC), it was corrected by applying the Heyd-Scuseria-Ernzerhof (HSE) screened-nonlocal-exchange functional of the generalized Kohn-Sham scheme. ${ }^{45}$

While performing all calculations, a plane-wave basis set with a kinetic energy cutoff of $400 \mathrm{eV}$ was used. For the convergence criterion, the total energy difference between the sequential steps in the calculations was taken to be $10^{-6} \mathrm{eV}$. The total force in the unitcell was reduced to a value of less than $10^{-5} \mathrm{eV} \AA^{-1}$. To hinder interactions between the adjacent cells, a vacuum spacing of at least $13 \AA$ was used along the $z$-direction. All calculations were performed with the spin polarized case in the consideration of spin orbit interactions. Analysis of the charge transfers in the structures was performed using the Bader technique. ${ }^{46}$ The vibrational properties were obtained via the PHONOPY code,${ }^{47}$ which uses the force constants calculated by a finite-displacement method.

\section{Structural, vibrational, and electronic properties of the Janus WSSe single layer}

As shown in Fig. 1(a), the crystal structure of the Janus WSSe single layer has a hexagonal symmetry and crystallizes in the $2 \mathrm{H}$ phase. The Janus WSSe structure consists of a middle $\mathrm{W}$ layer covalently bonded with surface chalcogenide $\mathrm{S}$ and Se layers. The crystal structure of WSSe has a hexagonal lattice and its lattice parameters, $|\vec{a}|=|\vec{b}|$, are found to be $3.25 \AA$. The bond lengths of $\mathrm{W}-\mathrm{S}$ and $\mathrm{W}-\mathrm{Se}$ in the Janus single layer are calculated to be 2.42 and $2.54 \AA$, respectively. The thickness of the Janus structure, which is defined to be the vertical distance between the uppermost and lowermost chalcogenide atoms, is calculated to be $3.23 \AA$. The ground state analysis of the Janus WSSe single layer reveals that the Janus structure is a nonmagnetic 2D material. In addition, Table 1 presents the structural properties and electronic band gap values of the Janus WSSe single layer in comparison with its tungsten analogs, $\mathrm{WS}_{2}$ and $\mathrm{WSe}_{2}$, single layers.

The phonon band dispersion diagram, as shown in Fig. 1(b), indicates the dynamical stability of the Janus WSSe single layer. There are 3 atoms within the primitive unit cell of the Janus
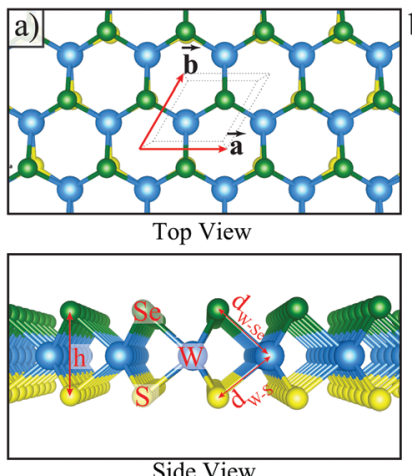

Side View

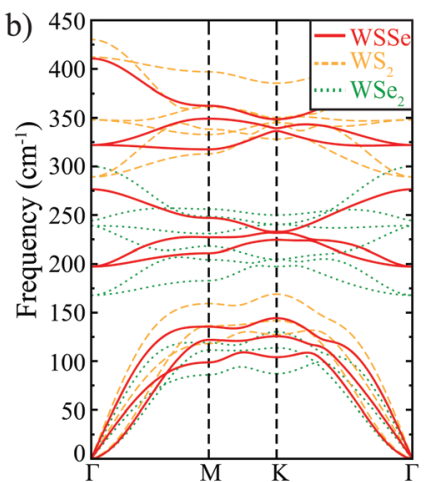

Fig. 1 (a) Top and side perspective views of the geometric structure of the Janus WSSe single layer. Turquoise, yellow and green balls show W, S and Se atoms, respectively. (b) Phonon band diagram of the Janus WSSe single layer (red line). Phonon band diagrams of the $\mathrm{WS}_{2}$ and $\mathrm{WSe}_{2}$ single layers are also shown as orange dashed lines and green dot lines, respectively.

Table 1 The calculated ground state properties of the single layer Janus structure of WSSe and its tungsten analog single layers, $\mathrm{WS}_{2}$ and $\mathrm{WSe}_{2}$ : the lattice parameter of the primitive unit cell, $a$; the distance between $W$ and

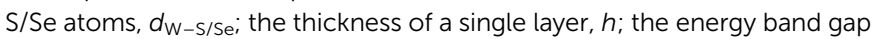
of the structure calculated within GGA + SOC + HSE06, $E_{\mathrm{g}}$

\begin{tabular}{llllll}
\hline & $a(\AA)$ & $d_{\mathrm{W}-\mathrm{S}}(\AA)$ & $d_{\mathrm{W}-\mathrm{Se}}(\AA)$ & $h(\AA)$ & $E_{\mathrm{g}}(\mathrm{eV})$ \\
\hline $\mathrm{WSSe}$ & 3.25 & 2.42 & 2.54 & 3.23 & 1.83 \\
$\mathrm{WS}_{2}$ & 3.18 & 2.41 & - & 3.13 & 2.01 \\
$\mathrm{WSe}_{2}$ & 3.33 & - & 2.55 & 3.34 & 1.61
\end{tabular}




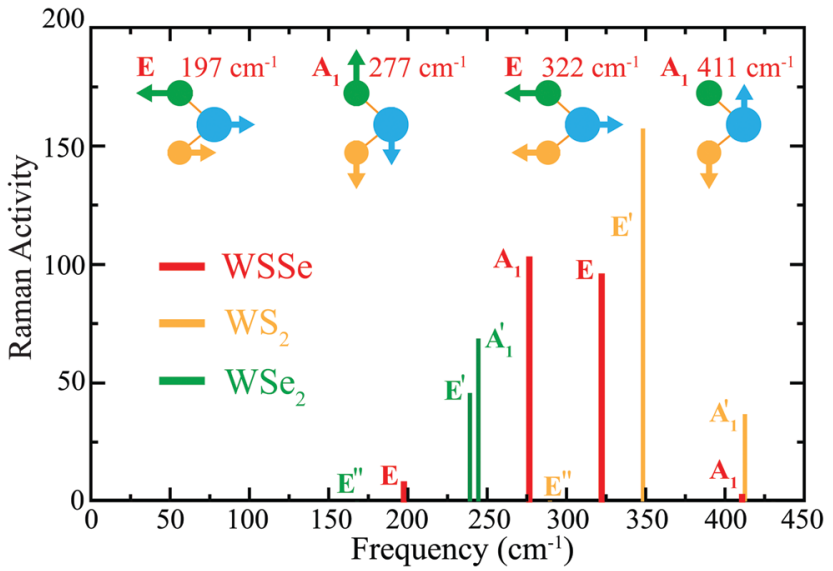

Fig. 2 Calculated Raman activities of the Janus WSSe single layer in comparison with those of the analog single layers. Additionally, schematic representations of the eigenvectors corresponding to the optical modes are shown

structure, thereby, its phonon spectrum includes 9 phonon branches, 3 acoustic and 6 optical. The 6 optical phonon branches consist of 2 singly-degenerate ( 277 and $411 \mathrm{~cm}^{-1}$ ) and 2 doubledegenerate (197 and $322 \mathrm{~cm}^{-1}$ ) vibrational modes. The phonon band diagram of the Janus single layer has a gap between the acoustic and optical regions, and also two gaps between the optical phonon branches, incontrast to the phonon band diagrams of single layers of $\mathrm{WS}_{2}$ and $\mathrm{WSe}_{2}$, as shown in Fig. 1(b). The distinctive optical phonon energies of the highest optical phonon mode may allow localization of phonons on one side of the Janus structure.

The eigenvectors and Raman activities correspond to the related optical modes as shown in Fig. 2. Moreover, the Raman activities of the modes of the WSSe single layer are also shown in comparison with those of the analog single layers. The Janus WSSe structure preserves the $C_{3 \mathrm{v}}$ point group symmetry; besides, a non-centrosymmetric structure exists in the Janus single layer. For this reason, $2 \mathrm{E}$ and $2 \mathrm{~A}_{1}$ optical modes are calculated as Raman active modes as well as IR-active. The prominent Raman active peaks that can be found in the Raman measurements appear at 277 and $322 \mathrm{~cm}^{-1}$, which are out-ofplane and in-plane optical modes, respectively.

The electronic band diagram of the Janus WSSe single layer is shown in Fig. 3(a). It is seen that the Janus structure is a direct band gap semiconductor and its valence band minimum (VBM) and conduction band minimum (CBM) reside at the $K$ symmetry point as $\mathrm{WS}_{2}$ and $\mathrm{WSe}_{2}$ single layers. The calculated energy band gap of the Janus WSSe single layer within GGA + SOC + HSE06 is found to be $1.83 \mathrm{eV}$. In addition to that, the energy band gaps of the $\mathrm{WS}_{2}$ and $\mathrm{WSe}_{2}$ single layers, are calculated to be 2.01 and $1.61 \mathrm{eV}$. Although there has been a debate on the band gap type of tungsten chalcogenides, ${ }^{48,49}$ the selected parameters in this work agree well with recently reported photoluminescence data and theoretical results. ${ }^{12,50}$

Construction of the electronic band diagram of the Janus structure is related to the tensile and compressive strains on the analogs of the Janus structure. Upon the formation of the WSSe single layer, the sulfide side experiences $2.2 \%$ tensile

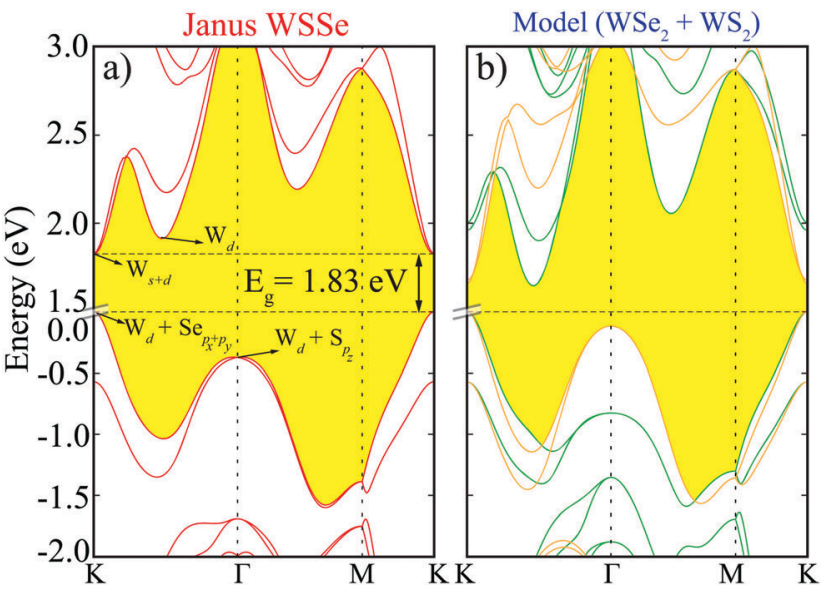

Fig. 3 (a) Electronic band diagram of the Janus WSSe single layer. (b) Superposition of the electronic band diagrams of the $2.2 \%$ tensile strained $\mathrm{WS}_{2}$ (orange color) and $2.3 \%$ compressive strained $\mathrm{WSe}_{2}$ (green color). Scissor operation is applied between 0 to $1.5 \mathrm{eV}$ in the $y$ axis.

strain, whereas the selenide side experiences $2.3 \%$ compressive strain. Fig. 3(b) shows the superposition of the electronic band diagrams of the strained $\mathrm{WS}_{2}$ and $\mathrm{WSe}_{2}$ single layers. Consistent with previous theoretical and experimental work, ${ }^{35}$ negligible change in the VBM and CBM points of the $\mathrm{WS}_{2}$ single layer is found under tensile strain and the structure is still a direct band gap semiconductor. On the other hand, the compressive strain on the $\mathrm{WSe}_{2}$ structure changes the CBM points to within the $K-\Gamma$ point from the $K$ symmetry point and makes it an indirect band gap semiconductor. It is found that the $\mathrm{p}_{x}+\mathrm{p}_{y}$ orbitals of Se contribute the VBM; for this reason, the selenide side is responsible for the VBM at the $K$ point. As expected from the superposition sketch, the effect of the sulfide side in the VBM is seen as the appearance of the $\mathrm{p}_{z}$ orbital of $\mathrm{S}$ at the $\Gamma$ point. Additionally, the d orbitals of $\mathrm{W}$ contribute CBM and VBM points all over the $k$-space, whereas only the s orbitals of $\mathrm{W}$ appear at the $\Gamma$ point to contribute the CBM. It is known that the workfunction of $\mathrm{WS}_{2}$ is higher than that of $\mathrm{WSe}_{2}$ and the Janus structure includes both surfaces; therefore, Fermi level shifting is constituted rather than Fermi level alignment in the Janus type structures. ${ }^{51}$ Consequently, the Fermi level shifting of the strained electronic band diagrams in order to adhere to the electron affinity rule constructs the Janus electronic band structure. Owing to hetero-chalcogenide surface layers, the surfaces of the Janus single layer have different workfunction values, and hence the Janus type is unique not only due to its electronic behavior, but also for its possible bilayer structures.

\section{Bilayer Janus WSSe structures}

\section{A Stacking types and energetics}

In this section, we discuss the ground state stacking orders and the phononic characteristics of the bilayers of the Janus WSSe crystals. 5 possible stackings, ${ }^{52} \mathrm{AA}, \mathrm{AA}^{\prime}, \mathrm{A}^{\prime} \mathrm{B}, \mathrm{AB}^{\prime}$, and $\mathrm{AB}$, are studied for the bilayer of Janus structure. Fig. 4 shows the possible stackings of the bilayer of Janus WSSe structure, in 

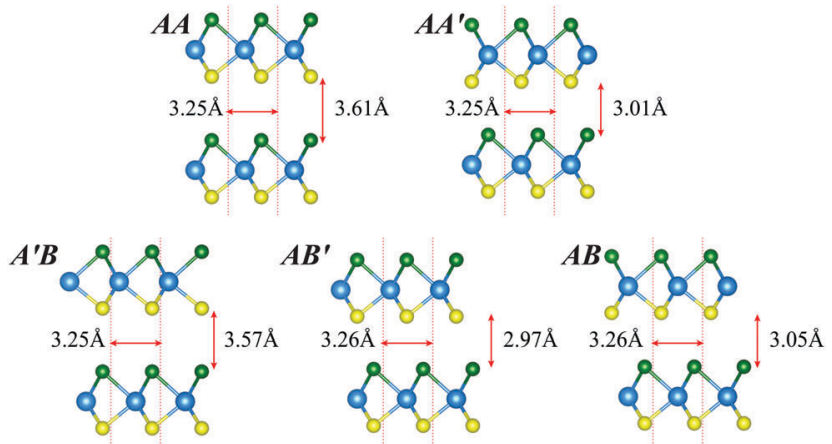

Fig. 4 Possible stacking orders of the bilayers of Janus WSSe structure. Schematic representations are only illustrated for stackings of the $\mathrm{S}-\mathrm{Se}$ junction bilayer of WSSe. Two structural parameters, lattice constant and layer-layer distance, of the bilayers of WSSe are also shown.

addition to the lattice parameters and layer-layer distances of the possible bilayers of WSSe.

In the Janus structure, the bilayer stacking orders are found to be in three different junctions due to different chalcogenide atoms at the interface, which are S-Se, S-S and Se-Se junctions. The lattice parameters of the bilayers of WSSe show negligible change for all stacking and junction types. On the other hand, the layer-layer distances of the bilayers of Janus structure can be used as distinctive structural features. Two distinguishable sub-stacking groups are found in the bilayers of Janus WSSe structure. $\mathrm{AA}$ and $\mathrm{A}^{\prime} \mathrm{B}$ form one sub-group, in contrast, $\mathrm{AA}^{\prime}, \mathrm{AB}^{\prime}$ and $\mathrm{AB}$ are part of the other sub-group. They are clearly distinguishable by their layer-layer distances, if the distance is higher than $3.50 \AA$, stacking order is found in one group, which consists of $\mathrm{AA}$ and $\mathrm{A}^{\prime} \mathrm{B}$ stackings. If the distance is smaller than $3.20 \AA$, the stacking order is in the other group. This assumption is valid for all junction variations; thus, the imaging techniques can be helpful to determine the stacking order of the bilayer. However, it is not possible to ascertain the junction type by utilizing information about layer-layer distances and the top side image of the bilayers of WSSe.

Moreover, the first-principles calculations showed that the Se-Se junction bilayer of Janus WSSe structure has the ground state structure for the bilayer of WSSe as seen in Table 2. The ground state stacking type of the bilayer of WSSe is found to be $\mathrm{AA}^{\prime}$ stacking. In addition, the energy difference between the $\mathrm{AA}^{\prime}$ and $\mathrm{AB}^{\prime}$ stacking orders is found to be $(9 \mathrm{meV})$ comparable to the thermal energy at room temperature $(25 \mathrm{meV})$, which indicates that it is possible to observe both stacking orders.

Table 2 The structural formation energies of the bilayers of Janus WSSe structure in comparison with different stackings. Energy of the ground state of the bilayer of WSSe is taken as zero, and the energies of the other states are set relative to the ground state energy as eV per formula unit. The ground state stacking of each junction type is shown in bold

\begin{tabular}{llllll}
\hline WSSe bilayer & $\mathrm{AA}$ & $\mathrm{AA}^{\prime}$ & $\mathrm{A}^{\prime} \mathrm{B}$ & $\mathrm{AB}^{\prime}$ & $\mathrm{AB}$ \\
\hline S-Se & 0.125 & 0.016 & 0.121 & $\mathbf{0 . 0 1 5}$ & 0.039 \\
S-S & 0.147 & 0.043 & 0.139 & $\mathbf{0 . 0 3 9}$ & 0.053 \\
Se-Se & 0.117 & $\mathbf{0 . 0 0 0}$ & 0.113 & 0.009 & 0.035
\end{tabular}

Regarding the S-Se and S-S junction types, the first- and second-lowest energy stacking orders are found to be $\mathrm{AB}^{\prime}$ and $\mathrm{AA}^{\prime}$, respectively.

\section{B Monitoring the stacking type by phonons}

While Janus types of TMDs are possible to synthesize, the detection of the stacking order of multi-layered Janus TMDs is quite difficult and needs further investigation. Therefore, it is essential to predict the characteristics of the different stackings in the multi-layered Janus TMDs. In this section, we discuss the vibrational properties of the ground state stacking of the bilayers of Janus WSSe structure.

The phonon band diagrams of the S-Se, S-S and Se-Se junction bilayers are presented in Fig. 5(a). It is seen that the ground state structures of the bilayers are dynamically stable as well, and hence all junction types for the bilayers of WSSe are possible. Correlatively with the Janus WSSe single layer, the phonon band structures of the bilayers of WSSe are roughly comparable and have 3 gaps between the 4 phonon band branch regions, which are acoustic regions including shear mode (I) and layer breathing mode (II), bottom optical region (III, IV, V and VI), mid optical region (VII and VIII), and top optical region (IX and X). The gap between the acoustic region and the bottom optical region is about $50 \mathrm{~cm}^{-1}$ and similarly, the gap between the bottom optical region and the mid optical region is about $40 \mathrm{~cm}^{-1}$. Likewise, both gaps show negligible difference in the bilayers of Janus WSSe structure. However, the gap between the mid optical region and the top optical region at the $\Gamma$ point is large, but the gap at the $K$ point in the phonon band diagram is small and shows a difference between the bilayers of WSSe. Besides, in the S-Se junction structure, the VII and VIII modes of the mid optical region cross at the $K$ symmetry point, whereas the other structures do not.

According to the junction types, the phonon branches that have similar eigenvectors split differently in the bottom optical, mid optical and top optical regions of the phonon band diagrams at the symmetry points. At the top optical region, the S-S and S-Se junction bilayers show splitting in eigen-frequencies, whereas the Se-Se junction bilayer shows no splitting. On the other hand, the Se-Se junction bilayer shows eigen-frequency splitting at the mid optical region, leastwise, the S-Se junction bilayer also shows splitting; in contrast, the S-S junction bilayer shows ignorable splitting in eigen-frequencies. Two reasons are considered to be a determination for the splitting behaviors of the different junction stackings of the bilayers of WSSe. Firstly, eigenvectors at the interface vibrate more than eigenvectors at the surface, and secondly, the $\mathrm{S}$ atom binds the transition metal atom stronger than the Se atom. Eventually, splitting at the optical phonon modes of the bilayers of WSSe are related to the vibrational characteristics of the chalcogenide atoms and the position of the chalcogenide atoms in the Janus crystal.

In addition to the vibrational spectra of the bilayers of the Janus crystal, Raman activities of the optical modes are investigated. To provide a clear visualization of the shift of eigen-frequencies, we divide the frequency space into 6 domains, as shown in Fig. 5(b). Moreover, the characteristic atomic motions of the optical modes 

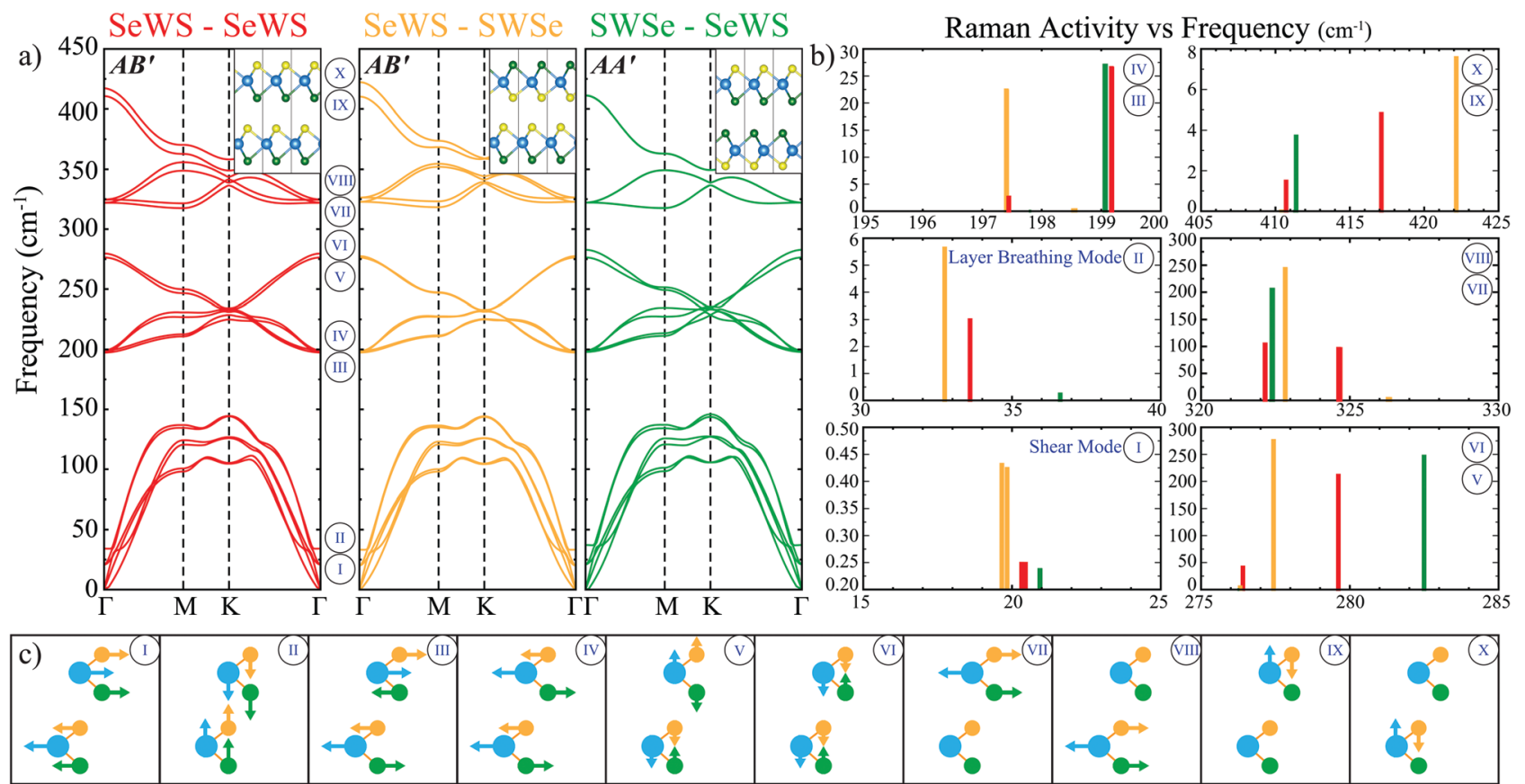

Fig. 5 (a) Phonon band diagram of the S-Se, S-S and Se-Se junction bilayer of Janus WSSe ground state structures, respectively. (b) Raman activities of the optical modes of the bilayers of Janus WSSe structure. (c) Schematic representation of the eigenvectors of the enumerated optical phonon modes for the S-Se junction bilayer of Janus WSSe structure.

of the bilayers of WSSe are shown in Fig. 5(c). Experimentally, it is possible to measure small frequency shifts as $1-2 \mathrm{~cm}^{-1}$ so that the Raman shifts in our findings are such as to be measurable.

The shear (I) and layer breathing (II) modes are special characteristic modes for bilayer structures and as all bilayer materials, ${ }^{53,54}$ the eigen-frequency of the shear modes is calculated to be lower than that of the layer breathing modes in the bilayers of the Janus crystal. Raman activities of the shear and layer breathing modes of the bilayers decrease from $\mathrm{S}-\mathrm{S}$ to the first S-Se and the second Se-Se junction, respectively, whereas the eigen-frequencies increase in the same order as 33, 34 and $37 \mathrm{~cm}^{-1}$, respectively. A slight frequency shift is calculated in shear modes of the ground state structure of the bilayers; however, distinctive Raman fingerprints for the bilayers are found in the layer breathing modes. Moreover, double the amount of shear mode Raman activity compared to the other junctions is another sign to distinguish the S-S junction bilayer.

The lowest optical modes of the bilayers of WSSe, III and IV, originate from in-plane vibrations and differ from each other due to the vibrations of layers that are counter-phase and in-phase, respectively. For all junction types, the eigen-frequencies of the III and IV modes show negligible difference and are $\sim 197.5$ and $\sim 199 \mathrm{~cm}^{-1}$, respectively. Nevertheless, the single intense peak at $\sim 199 \mathrm{~cm}^{-1}$ determines the Se-Se junction bilayer in the Raman activity. Besides, from the modes III to IV, stepdown in the Raman activity points out the S-S junction and vice versa for the S-Se junction bilayer.

In contrast to the optical modes III and IV, the V and VI modes originate from out-of-plane vibrations. The characteristic lattice vibrations of the V and VI modes are in comparison with the layer vibrations whether atomic motions in each layer are counter-phase or in-phase, respectively. The eigen-frequency for the $\mathrm{V}$ mode is the same for all bilayers of WSSe $\sim 276 \mathrm{~cm}^{-1}$; on the other hand, the Raman activity of the optical mode is the highest for the S-Se junction and the lowest for the Se-Se junction bilayer. Moreover, one of the two prominent peaks in the bilayers is seen in the VI mode and the highly Raman active optical mode makes it possible to differentiate the bilayers of Janus WSSe structure. The distinctive eigen-frequencies are $\sim 277,280$ and $283 \mathrm{~cm}^{-1}$ for the S-S, S-Se and Se-Se junction bilayers, respectively.

In contrast to all the abovementioned modes, the last four optical modes are the modes formed by opposite vibration directions between the $\mathrm{W}$ atoms and chalcogenide atoms in the Janus layers. The optical modes VII and VIII originate from the in-plane vibrations of the top and bottom single layer in the bilayer of Janus structure, respectively. The other group of the two prominent peaks is found in the VII and VIII mode of the bilayers of WSSe. The trend in both the eigen-frequencies and Raman activities of the optical modes of the bilayers of Janus structures shows a distinctive feature. Additionally, splitting in the eigen-frequencies, which is induced by difference between the interface atoms of the bilayer structures, strengthens the distinctive feature of the optical modes. The pair of prominent peaks between 320 and $325 \mathrm{~cm}^{-1}$ is the most visible fingerprint for the detection of the S-Se junction bilayer in the Raman measurement.

Similar to the VII and VIII modes, the IX and $\mathrm{X}$ optical modes originate from the vibrations of the top or bottom single layer in the bilayer of Janus structure, however; the vibrations 
are in the out-of-plane direction. In addition, atomic vibrations of the IX and X modes contain only the counter-phase motion of the transition metal $\mathrm{W}$ and chalcogenide $\mathrm{S}$ atoms. The eigenfrequencies of the $\mathrm{X}$ mode are 411,417 and $422 \mathrm{~cm}^{-1}$ for the Se-Se, S-Se and S-S junction bilayers, respectively, and a gradually increasing trend in the Raman activity is seen in the same order. Therefore, the Raman activities of the optical modes and difference in the eigen-frequencies of the bilayers is enough to distinguish the junction type. Consequently, all optical modes are calculated to be Raman active in the bilayers owing to the non-centrosymmetric feature in the Janus type structures.

The results obtained from both frequency shift and peak intensity present valuable fingerprints regarding the stacking order and junction type of the bilayers of Janus structure. Since the vibrational characteristics of $2 \mathrm{D}$ materials are easy to measure, the findings can be used to monitor the stacking orders and junction types of the bilayers of Janus crystals via Raman measurements.

\section{Conclusions}

In this study, we investigated the properties of dynamically stable single layer Janus WSSe structures and bilayers of WSSe. Due to the non-centrosymmetric structure of the Janus single layer, all optical modes were calculated to be Raman active and two prominent peaks at 277 and $322 \mathrm{~cm}^{-1}$ are likely to be observed in the Raman spectrum. Furthermore, the Janus WSSe single layer was calculated to be a direct band gap semiconductor due to its tungsten single layer analogs $\mathrm{WS}_{2}$ and $\mathrm{WSe}_{2}$. It was found that the structural and the electronic properties of the analog single layers determine the properties of Janus single layers.

In order to identify the ground state stacking type of the bilayers of Janus WSSe structures, we considered the total energies of the possible stacking orders that are seen in the bilayers of TMDs. $\mathrm{AA}^{\prime}$ and $\mathrm{AB}^{\prime}$ stacking types were found to be the favorable stacking orders for the bilayers of WSSe. It was shown that the Se-Se junction bilayer is the minimum energy configuration of the bilayers in which the Se atoms faced at the interface of the bilayers of WSSe.

In addition, it was also found that the $\mathrm{S}-\mathrm{S}$ and $\mathrm{S}-\mathrm{Se}$ junction types are also possible at the interface of the Janus bilayers due to their comparable total energies. Although it is not sufficient, it was seen that the thickness can be used to discriminate the energetically favorable bilayers. Moreover, the vibrational properties of the favorable bilayers of Janus WSSe structure were investigated. The phonon band structures of the favorable bilayers showed that splitting of the optical phonon branches is a distinctive feature to identify the junction types. It appears that both the frequency shifts and Raman activities of the optical modes of the bilayers offer particular opportunities to define the character of the compounds. The results presented here show that monitoring the stacking order and junction type of the bilayers of recently synthesized Janus crystals can be achieved by using Raman spectroscopy.

\section{Conflicts of interest}

There are no conflicts to declare.

\section{Acknowledgements}

Computational resources were provided by TUBITAK ULAKBIM, the High Performance and Grid Computing Center (TRGrid e-Infrastructure). HS acknowledges financial support from the TUBITAK under the project number 117F095.

\section{References}

1 K. S. Novoselov, D. Jiang, F. Schedin, T. J. Booth, V. V. Khotkevich, S. V. Morozov and A. K. Geim, Proc. Natl. Acad. Sci. U. S. A., 2005, 102, 10451.

2 H. Sahin, O. Leenarts, S. K. Singh and F. M. Peeters, WIREs Comput. Mol. Sci., 2016, 6, 351.

3 M. Xu, T. Liang, M. Shi and H. Chen, Chem. Rev., 2013, 113, 3766.

4 M. Chhowalla, H. S. Shin, G. Eda, L.-J. Li, K. P. Loh and H. Zhang, Nat. Chem., 2013, 5, 263.

5 H. Sahin, E. Torun, C. Bacaksiz, S. Horzum, J. Kang, R. T. Senger and F. M. Peeters, WIREs Comput. Mol. Sci., 2016, 6, 351.

6 X. Zhang, X.-F. Qiao, W. Shi, J.-B. Wu, D.-S. Jiang and P.-H. Tan, Chem. Soc. Rev., 2015, 44, 2757.

7 S. Manzeli, D. Ovchinnikov, D. Pasquier, O. V. Yazyev and A. Kis, Nat. Rev. Mater., 2002, 65, 125407.

8 R. A. Gordon, D. Yang, E. D. Crozier, D. T. Jiang and R. F. Frindt, Phys. Rev. B: Condens. Matter Mater. Phys., 2002, 65, 125407.

9 K. F. Mak, C. Lee, J. Hone, J. Shan and T. F. Heinz, Phys. Rev. Lett., 2010, 105, 136805.

10 J. N. Coleman, M. Lotya, A. O'Neill, S. D. Bergin, P. J. King, U. Khan, K. Young, A. Gaucher, S. De, R. J. Smith, I. V. Shvets, S. K. Arora, G. Stanton, H.-Y. Kim, K. Lee, G. T. Kim, G. S. Duesberg, T. Hallam, J. J. Boland, J. J. Wang, J. F. Donegan, J. C. Grunlan, G. Moriarty, A. Shmeliov, R. J. Nicholls, J. M. Perking, E. M. Grieveson, K. Theuwissen, D. W. McComb, P. D. Nellist and V. Nicolosi, Science, 2011, 331, 568.

11 Q. H. Wang, K. Kalantar-Zadeh, A. Kis, J. N. Coleman and M. S. Strano, Nat. Nanotechnol., 2012, 7, 699.

12 H. Sahin, S. Tongay, S. Horzum, W. Fan, J. Zhou, J. Li, J. Wu and F. M. Peeters, Phys. Rev. B: Condens. Matter Mater. Phys., 2013, 87, 165409.

13 J. S. Ross, P. Klement, A. M. Jones, N. J. Ghimire, J. Yan, D. G. Mandrus, T. Taniguchi, K. Watanabe, K. Kitamura, W. Yao, D. H. Cobden and X. Xu, Nat. Nanotechnol., 2014, 9, 268.

14 B. Chen, H. Sahin, A. Suslu, L. Ding, M. I. Bertoni, F. M. Peeters and S. Tongay, ACS Nano, 2015, 9, 5326.

15 A. Politano, G. Chiarello, C.-N. Kuo, C. S. Lue, R. Edla, P. Torelli, V. Pellegrini and D. W. Boukhvalov, Adv. Funct. Mater., 2018, 28, 1706504. 
16 B. Peng, P. K. Ang and K. P. Loh, Nano Today, 2015, 10, 128. 17 A. L. Elias, N. Perea-López, A. Castro-Beltran, A. Berkdemir, R. Lv, S. Feng, A. D. Long, T. Hayashi, Y. A. Kim, M. Endo, H. R. Gutierrez, N. R. Pradhan, L. Balicas, T. E. Mallouk, F. Lopez-Urias, H. Terrones and M. Terrones, ACS Nano, 2013, 7, 5235.

18 J.-K. Huang, J. Pu, C.-L. Hsu, M.-H. Chiu, Z.-Y. Juang, Y.-H. Chang, W.-H. Chang, Y. Iwasa, T. Takenobu and L.-J. Li, ACS Nano, 2014, 8, 923.

19 S. M. Eichfeld, L. Hossain, Y.-C. Lin, A. F. Piasecki, B. Kupp, A. G. Birdwell, R. A. Burke, N. Lu, X. Peng, J. Li, A. Azcatl, S. McDonnell, R. M. Wallace, M. J. Kim, T. S. Mayer, J. M. Redwing and J. A. Robinson, ACS Nano, 2015, 9, 2080.

20 Y. Gao, Z. Liu, D.-M. Sun, L. Huang, L.-P. Ma, L.-C. Yin, T. Ma, Z. Zhang, X.-L. Ma, L.-M. Peng, H.-M. Cheng and W. Ren, Nat. Commun., 2015, 6, 8596.

21 K. M. McCreary, A. T. Hanbicki, G. G. Jernigan, J. C. Culbertson and B. T. Jonker, Sci. Rep., 2016, 6, 19159.

22 C.-H. Lee, G.-H. Lee, A. M. van der Zande, W. Chen, Y. Li, M. Han, X. Cui, G. Arefe, C. Nuckolls, T. F. Heinz, J. Guo, J. Hone and P. Kim, Nat. Nanotechnol., 2014, 9, 676.

23 X. Hong, J. Kim, S.-F. Shi, Y. Zhang, C. Jin, Y. Sun, S. Tongay, J. Wu, Y. Zhang and F. Wang, Nat. Nanotechnol., 2014, 9, 682.

24 Y. Gong, J. Lin, X. Wang, G. Shi, S. Lei, Z. Lin, X. Zou, G. Ye, R. Vajtai, B. I. Yakobson, H. Terrones, M. Terrones, B. K. Tay, J. Lou, S. T. Pantelides, Z. Liu, W. Zhou and P. M. Ajayan, Nat. Mater., 2014, 13, 1135.

25 S. Tongay, W. Fan, J. Kang, J. Park, U. Koldemir, J. Suh, D. S. Narang, K. Liu, J. Ji, J. Li, R. Sinclair and J. Wu, Nano Lett., 2014, 14, 3185.

26 N. Huo, J. Kang, Z. Wei, S.-S. Li, J. Li and S.-H. Wei, Adv. Funct. Mater., 2014, 24, 7025.

27 J. H. Yu, H. R. Lee, S. S. Hong, D. Kong, H.-W. Lee, H. Wang, F. Xiong, S. Wang and Y. Cui, Nano Lett., 2015, 15, 1031.

28 Y. Gong, S. Lei, G. Ye, B. Li, Y. He, K. Keyshar, X. Zhang, Q. Wang, J. Lou, Z. Liu, R. Vajtai, W. Zhou and P. M. Ajayan, Nano Lett., 2015, 15, 6135.

29 C. Lee, H. Yan, L. E. Brus, T. F. Heinz, J. Hone and S. Ryu, ACS Nano, 2010, 4, 2695.

30 A. Molina-Sanchez and L. Wirtz, Phys. Rev. B: Condens. Matter Mater. Phys., 2011, 84, 155413.

31 A. Molina-Sanchez, K. Hummer and L. Wirtz, Surf. Sci. Rep., 2015, 70, 155413.

32 C. R. Zhu, G. Wang, B. L. Liu, X. Marie, X. F. Qiao, X. Zhang, X. X. Wu, H. Fan, P. H. Tan, T. Amand and B. Urbaszek, Phys. Rev. B: Condens. Matter Mater. Phys., 2013, 88, 121301.

33 R. Yan, J. R. Simpson, S. Bertolazzi, J. Brivio, M. Watson, X. Wu, A. Kis, T. Luo, A. R. Hight Walker and H. G. Xing, ACS Nano, 2014, 8, 986.
34 M. Thripuranthaka and D. J. Late, ACS Appl. Mater. Interfaces, 2014, 6, 1158.

35 Y. Wang, C. Cong, W. Yang, J. Shang, N. Peimyoo, Y. Chen, J. Kang, J. Wang, W. Huang and T. Yu, Nano Res., 2015, 8, 2562.

36 Y. C. Cheng, Z. Y. Zhu, M. Tahir and U. Schwingenschlögl, Europhys. Lett., 2013, 102, 57001.

37 Q.-F. Yao, J. Cai, W.-Y. Tong, S.-J. Gong, J.-Q. Wang, X. Wan, C.-G. Duan and J. H. Chu, Phys. Rev. B: Condens. Matter Mater. Phys., 2017, 95, 165401.

38 A.-Y. Lu, H. Zhu, J. Xiao, C.-P. Chuu, Y. Han, M.-H. Chiu, C.-C. Cheng, C.-W. Yang, K.-H. Wei, Y. Yang, Y. Wang, D. Sokaras, D. Nordlund, P. Yang, D. A. Muller, M.-Y. Chou, X. Zhang and L.-J. Li, Nat. Nanotechnol., 2017, 12, 744.

39 J. Zhang, S. Jia, I. Kholmanov, L. Dong, D. Er, W. Chen, H. Guo, Z. Jin, V. B. Shenoy, L. Shi and J. Lou, ACS Nano, 2017, 11, 8192.

40 D. Er, H. Ye, N. C. Frey, H. Kumar, J. Lou and V. B. Shenoy, Nano Lett., 2018, 18, 3943.

41 G. Kresse and J. Hafner, Phys. Rev. B: Condens. Matter Mater. Phys., 1993, 47, 558.

42 G. Kresse and J. Furthmuller, Phys. Rev. B: Condens. Matter Mater. Phys., 1996, 54, 11169.

43 J. P. Perdew, K. Burke and M. Ernzerhof, Phys. Rev. Lett., 1996, 77, 3865.

44 S. Grimme, J. Comput. Chem., 2006, 27, 1787.

45 J. Heyd, G. E. Scuseria and M. Ernzerhof, J. Chem. Phys., 2003, 118, 8207.

46 G. Henkelman, A. Arnaldsson and H. Jonsson, Comput. Mater. Sci., 2006, 36, 354.

47 A. Togo, F. Oba and I. Tanaka, Phys. Rev. B: Condens. Matter Mater. Phys., 2008, 78, 134106.

48 C. Zhang, Y. Chen, A. Johnson, M.-Y. Li, L.-J. Li, P. C. Mende, R. M. Feenstra and C.-K. Shih, Nano Lett., 2015, 15, 6494.

49 W.-T. Hsu, L.-S. Lu, D. Wang, J.-K. Huang, M.-Y. Li, T.-R. Chang, Y.-C. Chou, Z.-Y. Juang, H.-T. Jeng, L.-J. Li and W.-H. Chang, Nat. Commun., 2017, 8, 929.

50 H. Zeng, G.-B. Liu, J. Dai, Y. Yan, B. Zhu, R. He, L. Xie, S. Xu, X. Chen, W. Yao and X. Cui, Sci. Rep., 2013, 3, 1608.

51 A. Kandemir and H. Sahin, Phys. Rev. B: Condens. Matter Mater. Phys., 2018, 97, 155410.

52 J. He, K. Hummer and C. Franchini, Phys. Rev. B: Condens. Matter Mater. Phys., 2014, 89, 075409.

53 A. A. Puretzky, L. Liang, X. Li, K. Xiao, K. Wang, M. MahjouriSamani, L. Basile, J. C. Idrobo, B. G. Sumpter, V. Meunier and D. B. Geohegan, ACS Nano, 2015, 9, 6333.

54 L. Liang, J. Zhang, B. G. Sumpter, Q.-H. Tan, P.-H. Tan and V. Meunier, ACS Nano, 2017, 11, 11777. 